\title{
New Structure and Physical Characteristics of Intelligent Real-time Control on Optical Coating
}

\author{
Shijun Xu' ${ }^{1}$, Xiaoling Ren ${ }^{2}$, Chunmin Zhang ${ }^{3}$ \\ ${ }^{1}$ School of Science, Xi'an Technological University \& Xi'an Jiaotong University, Xi'an 710032, China \\ ${ }^{2}$ School of Computer, Xi' an Polytechnic University, Xi'an 710048, China \\ ${ }^{3}$ School of Science, Xi' an Jiaotong University, Xi'an 710049, China \\ xushijun000@sina.com
}

\begin{abstract}
Comparing general optical thin-film deposition systems, which present poor precision and automation level on monitoring film thickness, we proposed a new intelligent photoelectric-control-analysis coating system. The structure of the new system includes a four-light path photoelectric system matching with double-frequency modulation hardware equipment and a compound filteration-control system. The compound filterationcontrol system contains anti-disturbance circuit, analog circuits of multi-stage amplifier, symmetrical dual-lock-phase amplifier, digital division, anti-pulse-disturbance digital filter, linearization of digital display, algorithm of picking off singular data, and extremumjudgment algorithm. Experiments show that all functions of intelligent real-time monitoring film thickness are achieved. The physical characteristics, for example, the static and dynamic stabilities and the control thin-film thickness precision, are extremely increased. The resolution of digital display as per reflectivity is $0.02 \%$. The duplication, i.e., standard deviation of monitoring thinfilm thickness, is less than $0.50 \%$. The $\mathrm{S} / \mathrm{N}$ approaches to 1500 . The linearity of drift is very high, and the static drift ratio is close to $1.63 \% / \mathrm{h}$. In conclusion, the new system has advantages of the high control precision for thin-film thickness and the favorable static and dynamic stabilities.
\end{abstract}

Index Terms - Intelligent Control, Optical Detection, Thin-film, Four-light Beams, Precision.

\section{Introduction}

Accurate monitoring-controlling of the thickness is a key technique for deposition of optical thin-film ${ }^{1-4}$. In many fields on coating optical thin-film and monitoring spectrum characteristic or optical constants, the exploration and development in abroad groups are in the lead. For example, the coating equipments of Leybold corporation in Germany and Carl Zeiss corporation in Switzerland have a higher level on automatic control, the thin-film thickness instruments of Honglun in Japan have good monitoring and control functions ${ }^{5,6}$. By comparison, the monitoring coating process of domestic equipments almost depends upon fixing human eyes on display number or instrument needle, and upon analyzing their changes. Consequently, the manual operations with lots of experience inevitably and subjectivity lead to inferior duplication and low qualified product ratio. Also, the poor automation level means lower efficiency and higher labor intensity in coatings.

The general coating equipment with low precision and automation level on monitoring thin-film thickness, a new photoelectric control and analysis system was investigated.
The new system included a four-light path photoelectric system and a dual-lock-phase circuit system and a comprehensive digital processing-controlling-analysis system. The test results show that the static and dynamic stabilities are extremely increased, along with controlling precision in monitoring optical thin-film thickness.

\section{New Construction And Principles Of Intelligent Thin- Film Thickness Monitoring System}

The main methods of monitoring thin-film thickness primarily contain the PEEMM (photoelectric extrema monitoring method), the quartz crystal oscillation method, the scanning ellipsometer by synchronous rotation of polarizer and analyzer, and the wide spectrum monitoring method ${ }^{6-10}$, etc. Especially, in monitoring optical thin-film thickness, the application of PEEMM is more extensive than other methods. Monitoring optical thin-film thickness by photoelectric detection technology directly influences its reflectance or transmittance of thin-film.

The PEEMM principle is according to the interference theory of film in wave optics. While optical film-thickness, i.e. $n d$, changes by $\lambda / 4$, reflectivity $R$ or transmittivity $T$ of vertical incidence light appears with an extremum. By means of monitoring the extrema in a depositing process, the monitoring thickness of $\lambda / 4$ film series is realized. In the regions nearby the extremum point, the change defined as $\partial R / \partial(n d)$ or $\partial T / \partial(n d)$ is very small, and the unsteady factors (e.g. noise etc.) are relatively strong, as a result, it is very difficult to improve the duplication and precision of monitoring thin-film thickness. To this defect of principle, although many remedial measures have been proposed, such as the extremum-pointlater control technique (belonging to a hysteretic controlling) and the pre-deposited layer technique and the multistage control technique ${ }^{4,5}$, the quality of monitoring thickness is dependent on static and dynamic stability and sensitivity of the monitoring system. This stability and sensitivity are just the purpose of this work.

\subsection{Construction of the General System}

In the general system of monitoring thin-film thickness, a light beam from a light source is modulated with singlefrequency, and divided into two bunches by a beam splitter. One light beam is received and concerted into a lock-phase 
demodulation signal by the optoelectronic sensor. After other bunch penetrates the control slice, the light, which has some information of thin-film thickness variety, passes through a monochrometer and a photomultiplier, therefore a measurement electric signal is acquired. Firstly, the two signals are processed to a low-noise transmitted signal in filter circuit and lock-phase amplifier circuit. Secondly, the signal is displayed on a numeral monitor. Finally, the monitoring thickness is accomplished by watching and determining the display number.

\subsection{Defects of the General System}

In the general system, anti-interference ability and stability of monitoring thin-film thickness are poor, and these main reasons are as follows.

The construction in which the measurement beam directly projects upon the incidence slit of monochrometer makes the abilities of anti-vibration and excluding the stray light fairly weak. At last, the stability of the monitoring signal is affected.

After the signal is processed only in analog filter circuit without further digital filter, it is showed on numeral phototube. Therefore, the numeral display of monitoring signal has bad stability.

The stabilities of light source and photomultiplier and their power supplies affect remarkably the stability of monitoring signal. For example, an infinitesimal fluctuation of high voltage of photomultiplier will cause an enormous output fluctuation that exceeds an input stage one order of magnitude, the luminous intensity of light source directly effects measuring beam intensity. In the monitoring system, consequently, very high demands of these stabilities and antiinterference abilities have been put forward.

\subsection{Structure of the New System}

As shown in Figure 1, a light beam from a source is modulated with double-frequency. Two different lock-phase demodulated signals of reference path and measuring path are gained with two silicon-photo battery, at the same time, a reference light beam and a measuring light beam are also obtained. The reference light beam transmitted with an optical fiber is directly received by a monochrometer and a photomultiplier. As well as, the measuring light beam, which is reflected on the control slice and contains some information of thin-film thickness variety, is received by the same optical fiber and the identical monochrometer and the alike photomultiplier. The superimposition of reference signal and measuring signal is separated, and processed in dual-lockphase amplifier circuit for digital acquisition, filtering procedure and division operation. A high quality signal including information of thin-film thickness variety is then acquired and displayed on numeral phototube. Moreover, the function of judgment thin-film thickness is realized with starting the judgment procedure and a servo system.

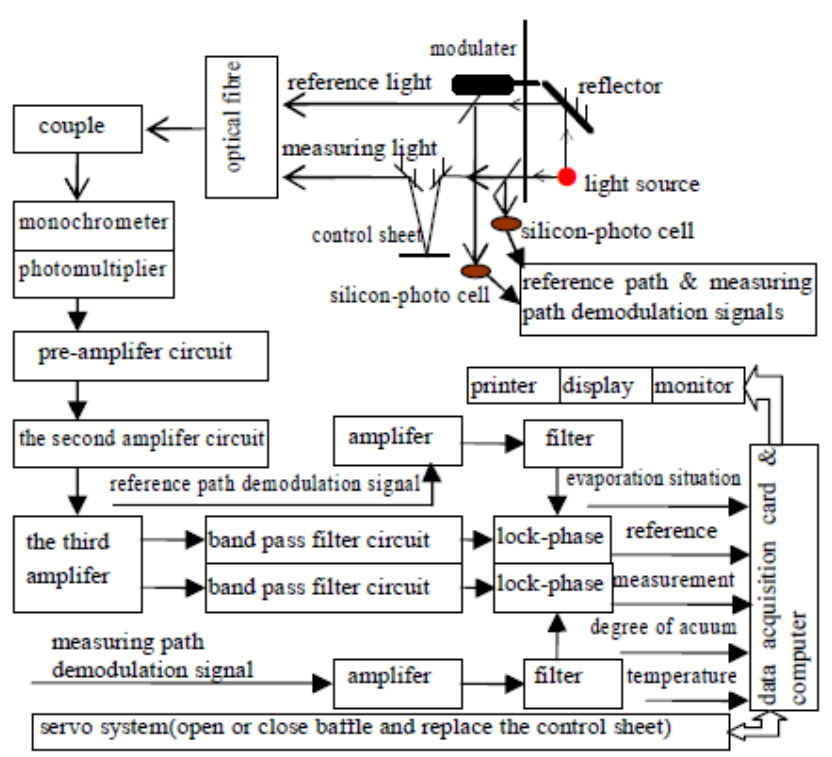

Fig.1. Structure Sketch of Proposed System.

\section{Novel Techniques Of New Thin-Film Thickness Monitoring System}

Compared with the general system, the novel technical characteristics are as follows.

\subsection{Double-frequency Modulator \& Four-light Path}

Optical modulation has many effects as follows. To measurement result, influence of the stray light is reduced, the bad effect of photomultiplier dark current is eliminated. The modulation signal provides the common work signal to lockphase amplifier and select-frequency amplifier.

For the general deposition equipment (for example, $D M D$ $700 \mathrm{~J}$ made in Peking Instrument Factory of China), the proposed system changes single-frequency modulator into double-frequency modulator, $33 \mathrm{~Hz}$ measuring light path and $383 \mathrm{~Hz}$ reference light path thus come into being. Besides, the double-frequency modulator is a basis of realizing four-light path system, dual-lock-phase circuit system and digital division (measuring data by reference data). Thus some common interference of light source, modulation motor, monochrometer, photomultiplier, etc., is extremely repressed.

The four-light path system includes $33 \mathrm{~Hz}$ modulated measuring light path taking some information of thin-film thickness variety, $383 \mathrm{~Hz}$ modulated reference light path with some information of light source modulated, $33 \mathrm{~Hz}$ and $383 \mathrm{~Hz}$ two modem light paths which are used for a dual-lock-phase amplifier circuit of cross correlation detection. In the original system, measuring light path and demodulation light path modulated with single-frequency, and this construction makes the stability of measuring signal remarkably hinging upon the stabilities of light source and photomultiplier and all power supplies. To resolve the problem, the measurement path and reference path are employed in the proposed system. The signals from two paths have the common fluctuation and interference of light source and photomultiplier. If the signals 
are divided, all this common fluctuation and interference should be cleared up. Although the four-light path is more complicated than single optical path of the original system, the work stability demands of these key equipments are greatly reduced, furthermore, the static stability of monitoring control system and the duplication of coatings are extremely improved.

\subsection{The Same Photomultiplier \& Monochrometer \& Optical Fiber}

The optical fiber is connected to the monochrometer for transmitting the light in the proposed system so that the utilization of optical energy is increased, while the measuring beam is directly projected upon the monochrometer slit in the original system. To the transmittal optical signal, the interference of the stray light can be eliminated. Particularly, the connection of optical fiber with monochrometer can eliminate the influence of coating equipment vibration to the measuring signal.

We transmit the measuring light and the reference light by a same set of optical fiber, an identical monochrometer and an alike photomultiplier. This design differed from two sets of monochrometers and photomultipliers can greatly reduce the difference mode interference, and is of benefit to the repression of the common mode interference in later division operation.

\subsection{Characters and Optimization Scheme on Signals}

In the thin-film deposition system with four-light beam, the light emitted from a light source will be split, and then modulated with dual-frequency, respectively. Through two silicon photo cells, the phase-lock demodulation signals of the reference and test circuits are obtained, and at the same time, the reference and test light beams are also obtained. The test light-beam with the information of the thickness of the thinfilm and reference light-beam are transmitted and converted by the same optical fiber, monochromator and photomultiplier tube. The superposition of the reference signal and test signal is separated through the amplifier and lock-phase circuits, and then, they are acquired by the data acquisition card. At last, the whole functions of controlling of the thin-film thickness are realized with the methods of high precision digital processing and the program controlling.

To achieve high stability and precision for the detection of the weak monitoring signal and automatic control of the thinfilm deposition, analog circuits with high precision and strong anti-disturbance combined with stable and efficient digital processing and controlling system must be designed and optimized. The core of the circuit detection system is the lockin amplifier8. The digital processing system is composed of the hardware of the computer, the digital tube, and the servo device and the software of high-precision digital processing. The digital processing system should complete the signal acquisition, physical explanation (calibration), eliminating the disturbance data, analyzing and calculating the intrinsic characters of the data.
Based on the four-light beams, dual-frequency modulation of the photoelectricity-extremum method, the weak signal output of the photoelectric sensor is handled multiply. The signal first passes through the multi-stage amplifier circuit, and then, it is detected by a symmetrical dual-lock-phase amplifierwith high precision. At last, the reference and test signals are processed and controlled by the computer after data acquisition. The right side of the dashed box represents the digital processing and controlling analysis system. The smoothing of the scanning data is handled after the monitoring of the thin-film deposition, and other processing and controlling are handled in real-time.

\subsection{The Dual-lock-phase Amplifier}

The dual-lock-phase amplifier circuit, without the singlelock-phase amplifier, is corresponding to above four-optical path system. The pre-amplifier and lock-phase circuit are designed with a symmetric arrangement, and chooses high and similar performance components. The above measures create some favorable conditions for later repressing the circuit drift with "digital division operation".

\subsection{Compound Digital Filter \& Digital Division \& Numeral Display}

For further reduction of differential-mode interferences from stray light in vacuum chamber and noise jamming in circuit etc, a compound digital filter technique is primarily adopted. In comparison with analog filter circuit, the technique has many advantages: only one filter program for many data channels, no hardware, reliable stability, lower frequency signal can be filtered, and the filtering parameters can be changed conveniently.

The measuring signal is digitally divided by the reference signal, which has higher accuracy and stability than the analog division. Especially, the digital division can eliminate the common mode interference from light source and photomultiplier etc. The ultimate purpose of the four-light path system and the dual-lock-phase amplifier circuit will be realized, and the stability of monitoring system should be raised.

After the film thickness signal is handled by the dual-lockphase amplifier circuit and the comprehensive digital processing-analysis technique (compound digital filter, digital division, linearization of numeral display), it is finally showed in numeral phototube. This technological stability is very high by contrast to the numeral display only carried in analog circuit in the general system.

\section{Experiment and Test}

Our experiment scheme include testing, contrasting, analyzing proposed system and original system, i.e., the static stability and the dynamic monitoring characteristics of the two systems are respectively tested, analyzed, compared on the $D M D-700 J$ deposition equipment.

The data of monitoring system are recorded and respectively fit with a linearity expression and a quadric polynomial expression. After the two expressions are 
evaluated with RSE (residual standard error) and CCC (composite correlation coefficient) and amended CCC, the accurate static drift law is got. While the numeral show of proposed system is linearly demarcated with quadric polynomial expression, the demarcated result is analyzed by variance and correlation coefficient, and the show sensitivity of the proposed system is determined. For four kinds of film series, which are $\mathrm{ZnS}$ and $\mathrm{MgF}_{2}$ single-layer and poly-layer optical thin-film, we have proceeded duplicate-coating experiments for 10 times in the two systems, respectively, guaranteeing the completely same technological conditions to the same film series in each experiment. Using the varianceanalysis method and uncertainty-analysis method, we have studied all monitoring data and spectrum-test data of each kind of film series, and got many quantitative parameters of film thickness.

Table.1. Static Properties of Two Systems.

\begin{tabular}{|l|l|c|l|l|}
\hline category & $S_{\mathrm{R}}$ & $\hat{\boldsymbol{\beta}}_{[\% / \mathrm{h}]}$ & $S_{\mathrm{r}}$ & $l$ \\
\hline general system & 0.00034017 & -14.7 & 0.00025774 & \pm 4.5 \\
\hline proposed system & 0.00000011 & -1.63 & 0.00000191 & \pm 1 \\
\hline
\end{tabular}

\section{Results}

The fundamental monitoring functions of the whole system have realized, which include the four-light path lens imaging system, the dual-lock-phase amplifier circuit system, the digital data acquisition-filter, the numeral display of analog signal, and the final automatic control. Thus the research lays a solid foundation for the next step product developments. The specific research conclusions are as follows.

The static drift characteristic of $D M D-700 J$ has been found as follows

$$
\hat{y}=5.02322 \times 10^{-8} x^{2}-0.000055 x+0.95836
$$

where Eq.(1) provides a mathematical amended model for automatic monitoring thin-film thickness, as well as gives a basis for analyzing influences of film spectrum from extremum-point-later control value.

The revised variance analysis method by the author, is applied to the analysis of coating stability or deposition duplication level and control precision. Some detailed quantitative parameters are given. All this results provide a basis for design and experimental operation of coating film.

In the proposed system, the numeral display properties have four aspects. First, the experimental results show that the signal-noise ratio approaches 1300 . Second, the linearity of numeral display is very high $(\mathrm{d} S / \mathrm{d} R \approx 1)$ and the residual deviation is very minor. This means that it can certainly be used as the photometer. Thirdly, the display-resolving limit of low reflectivity film, $\Delta R$, is equal to or more than $0.02 \%$. Lastly, in the all reflectivity scope, the display sensitivity of responding to reflectivity is higher, while the lower reflectivity corresponds to the higher show sensitivity.
Considered a low reflectivity case, many static properties of the two systems are listed in Table 1. Firstly, according to the $S_{\mathrm{R}}$ (regression deviation sum), it has been found that the linearity of monitoring drift is greatly higher in the proposed system than the original system, particularly, for the low reflectivity case, this conclusion provides some conveniences for experimental control and operation. Secondly, based on $\hat{\beta}$ (drift coefficient), the drift ratio of low reflectivity is very tiny and approaches 0 or $-1.63 \%$. In general, drift ratio in the proposed system is smaller than the old one. Thirdly, compared $S_{\mathrm{r}}$ of the two residual deviation sums, it can be seen that the static stability in the proposed system is better than the old system. To the high reflectivity film, the static stability is usually not only about 2 times higher than the original, but also further higher to the low reflectivity film. The lowest digit-flutter-scope $l$ means an uncertainty scope of the numeral display. The uncertainty of new system is obviously tiny, that is approximately $\pm 1, \pm 2, \pm 3.5$, whereas the original system is \pm 4.5 .

Dynamic properties of proposed system have been tested and various film series have been successfully monitored. The system's anti-interference ability has been greatly enhanced. For the single layer film of $\mathrm{ZnS}$, the comparison of monitoring stability or duplication of the proposed system and old system is as shown in Table 2 .

Table. 2. Stability or duplication of two systems.

\begin{tabular}{|c|c|c|c|}
\hline category & degree of remarkable difference & $S_{\mathrm{t}}$ & $S_{\mathrm{f}}$ \\
\hline general system & $191>\mathrm{F}_{0.01}(9,10)=4.94$ & 0.0874 & $1.25 \%$ \\
\hline proposed system & $102>\mathrm{F} 0.01(6,7)=7.19$ & 0.0562 & $0.50 \%$ \\
\hline
\end{tabular}

The degree of remarkable difference is defined consistency analysis of variance, which means the more obvious difference between the calculated value and the critical value and the poorer consistency. $S_{\mathrm{t}}$ is defined standard deviation of transmissivity and the standard deviation of thin-film thickness $\left(S_{\mathrm{f}}\right)$ is an object relativity index of duplication. Because $S_{\mathrm{f}}$ and $S_{\mathrm{t}}$ are equal to $0.50 \%$ and 0.0562 respectively, obviously, the duplication of proposed system is perfect.

\section{Conclusions}

The thin-film thickness standard deviation of the proposed system, which indicates duplication of monitoring thin-film thickness, is less than $0.50 \%$. The display resolving limit on reflectivity is $0.02 \%$. The signal-noise ratio approaches 1500 . The linearity of drift is very high, and the static drift ratio approaches $1.63 \% / \mathrm{h}$. In a word, based on higher sensitivity and stability of the proposed system, the static and dynamic stabilities or duplication are extremely raised, along with controlling precision in monitoring optical thin-film thickness.

\section{Acknowledgments}

Authors express heartfelt thanks to Jun Han professor of Xi'an Technological University for his help of the 
experiments. This work was sponsored by the Scientific Research Fund from Shaanxi Province Education Committee (No.06JK283) and supported in part by a grant from the National Natural Science Foundation of China (No.F051205).

\section{References}

[1] J. F. Tang and P. F. Gu, Editor, Thin-film Optics and Technology, Machinery Industry Press, Beijing (1989).

[2] N. Kaiser, T. stenfeigl and O. Stenzel, Opt. Precision Eng. 13, 2(2005).

[3] D. M. Hong, Z. Zhu and W. Yue, Opt. Precision Eng. 13, 2(2005).

[4] H. A. Macleod, Appl.Opt. 20, 1(1981).
[5] K. Tami and Y. Kiyoshi, Appl.Opt. 29, 34(1990).

[6] S. Xu, The Development of Real-time Monitoring System of Optical Thin-film Thickness, Dissertation of Xi'an Institute of Technology, Xi'an(2002).

[7] H. Ehlers, T. Grob and M. Lappschies, Opt. Precision Eng. 15, 2(2007)

[8] P. Bulkin, D. Daineka and D. Kouznetsov, Applied Physics 28, 2(2004).

[9] F. Benedic, P. Bruno and Ph. Pigeat, Applied Physics Letters 90, 7(2007).

[10] M. Navarro and J. Fandino, Surface Engineering 16, 1(2010). 